\title{
Identification of hub genes, key miRNAs and potential molecular mechanisms of colorectal cancer
}

\author{
SHASHA WU ${ }^{1}$, FEIXIANG WU ${ }^{2}$ and ZHENG JIANG ${ }^{1}$ \\ Departments of ${ }^{1}$ Gastroenterology, and ${ }^{2}$ Urology, The First Affiliated Hospital of \\ Chongqing Medical University, Chongqing 400016, P.R. China
}

Received March 23, 2017; Accepted August 17, 2017

DOI: 10.3892/or.2017.5930

\begin{abstract}
Colorectal cancer (CRC) is the most common cancer of the digestive system. The aim of the present study was to identify the potential biomarkers and uncover the underlying mechanisms. The gene and miRNA expression profiles were obtained from GEO database. The differentially expressed genes (DEGs) and miRNAs (DE miRNAs) were identified by GEO2R. The Gene Ontology (GO) enrichment and Kyoto Encyclopedia of Genes and Genomes (KEGG) pathway analysis were performed by KOBAS 3.0. The protein-protein interaction (PPI) network and miRNA-gene network were constructed by Cytoscape software. Then, the identified genes were verified by quantitative real-time PCR in both CRC tissue samples and cell lines. A total of 600 upregulated DEGs, 283 downregulated DEGs, 13 upregulated DE miRNAs and 7 downregulated DE miRNAs were identified. GO analysis results showed that upregulated DEGs were significantly enriched in binding, organelle and cellular process. Downregulated DEGs were enriched in binding, extracellular region and chemical homeostasis. KEGG analysis showed that the DEGs were mostly enriched in cell cycle and pathways in cancer. A total of eight genes were identified as biomarkers, including CAD, ITGA2, E2F3, BCL2, PRKACB, IGF1, SGK1 and NR3C1. Experimental validation showed that seven of the eight identified genes had the same expression trend as predicted, except for ITGA2. Besides, hsa-miR-552 and hsa-miR-30a were identified as key miRNAs. The present
\end{abstract}

Correspondence to: Dr Zheng Jiang, Department of Gastroenterology, The First Affiliated Hospital of Chongqing Medical University, 1 Youyi Road, Chongqing 400016, P.R. China

E-mail: jiangz1753@163.com

Abbreviations: CRC, colorectal cancer; DEGs, differentially expressed genes; DE miRNAs, differentially expressed genes miRNAs; GO, Gene Ontology; KEGG, Kyoto Encyclopedia of Genes and Genomes; PPI, protein-protein interaction; STRING, Search Tool for the Retrieval of Interacting Genes; MCODE, molecular complex detection; qPCR, quantitative real-time PCR

Key words: bioinformatics analysis, colorectal cancer, differentially expressed gene, protein-protein interaction, microRNA regulatory network study provides a series of biomarkers and mechanisms for the diagnosis and therapy of CRC. We also prove that although bioinformatics analysis is a wonderful approach, experiment validation is necessary.

\section{Introduction}

CRC is the most common digestive system cancer and is the third most common cancer in the world. Early stage CRC does not have obvious symptoms and is often diagnosed in advanced stage. CRC patients without metastases can be cured by surgery, but patients with advanced CRC are mainly treated with chemotherapy (1). Therefore, early diagnosis and treatment of CRC is needed.

Genetic changes have been investigated in CRC. Some significant oncogenes and tumor suppressor genes such as APC, KRAS and p53 are mutated in a considerable part of CRCs (2). This could allow screening and diagnosis of earlystage CRC, which may lead to substantial decreases in the mortality rate of CRC (3).

miRNAs are a series of small, non-coding regulatory RNAs. By repressing their target mRNAs, miRNAs regulate many cellular pathways, such as proliferation, apoptosis and differentiation. This feature of miRNAs provides a novel approach for cancer therapy (4). miRNAs such as miRNA-92a and miRNA-21 have shown significant connection with CRC, and can serve as biomarkers (5). $\mathrm{Ng}$ et al (6) reported plasma miR-92a can effectively discriminate CRC from control subjects. It has been verified that miR-21 can repress the tumor suppressor gene and induce cell invasion (7).

Microarray is a multiple lab-on-a-chip. To identify the biomarkers of CRC, we downloaded the gene and miRNA expression profiles of CRC from GEO database. Expression differences were compared between CRC tissues and normal colorectal tissues. By analyzing GO (8) and pathway enrichment (9) and constructing PPI network (10) and miRNA-gene network, we aimed to find key genes and miRNAs which play significant roles in the occurrence and development of CRC and discover new biomarkers for diagnosis and therapy.

\section{Materials and methods}

Microarray data. Three gene expression profiles (GSE21815, GSE32323 and GSE44076) and two miRNA expression 
profiles (GSE39845 and GSE53592) were obtained from GEO database (http://www.ncbi.nlm.nih.gov/geo/) (11). The GSE21815 datasets contained 132 CRC samples and 9 normal samples. GSE32323 included 17 CRC samples and 17 normal samples. GSE44076 consisted of 98 CRC samples and 98 normal samples. The miRNA expression profile of GSE39845 contained 3 CRC samples and 3 normal tissue samples. GSE53592 included 3 CRC samples and 3 normal samples.

Identification of DEGs and DE miRNAs. GEO2R (http:// www.ncbi.nlm.nih.gov/geo/info/geo2r.html) is an interactive web tool for comparing two or more groups of samples in a GEO series to identify DEGs or DE miRNAs across experimental conditions. We used GEO2R to identify DEGs and DE miRNAs. The P-value $<0.05$ and $\mid \log F C l>1$ were chosen as cut-off criteria.

Functional enrichment analysis of DEGs. KOBAS 3.0 (http:// kobas.cbi.pku.edu.cn/) is a latest updated web server for gene/ protein functional annotation and functional sets enrichment of gene (12). The GO enrichment and KEGG (13) pathway analysis were performed by KOBAS 3.0 online tool. In addition, $\mathrm{P}<0.05$ was set as the cut-off criterion.

PPI network construction and module analysis. To explore the interactive relationships among the DEGs, PPI network was constructed by the Search Tool for the Retrieval of Interacting Genes (STRING, version 10.0, http://string.embl.de/) and combined score $>0.4$ was set as the cut-off criterion. Then, PPI network was visualized by Cytoscape software (14). The Molecular Complex Detection (MCODE) app was performed to analyze modules of PPI network. MCODE scores $>3$ and the number of nodes $>4$ were set as cut-off criteria. The pathway enrichment analysis of genes in the modules was performed by KOBAS 3.0. $\mathrm{P}<0.05$ and input number $>3$ were considered to be significant.

miRNA-gene network construction. The target genes of DE miRNAs were predicted by five established miRNA target prediction programs (miRanda, MirTarget2, PicTar, PITA and TargetScan). The genes predicted by at least three programs were chosen as the targets of DE miRNAs. Then, miRNA-gene network was constructed. To identify the key gene biomarkers, we combined both PPI and miRNA-gene network. Genes with degree $\geq 20$ in PPI network and degree $\geq 3$ in miRNA-gene network were selected as gene biomarkers.

Cell culture. The human CRC cell lines HCT116 and HT-29 were obtained from the American Type Culture Collection Cell Center and cultured in RPMI-1640 medium (HyClone Laboratories, Inc., Logan UT, USA) supplemented with $10 \%$ fetal bovine serum (FBS; PAN-Biotech, Aidenbach, Germany) and $1 \%$ penicillin-streptomycin (Beyotime Institute of Biotechnology, Haimen, China) at $37^{\circ} \mathrm{C}$ in $5 \% \mathrm{CO}_{2}$.

Patient samples. CRC tissue samples and normal colorectal tissue samples were collected from patients with CRC in The First Affiliated Hospital of Chongqing Medical University (Chongqing, China). Ethics approval was obtained from the
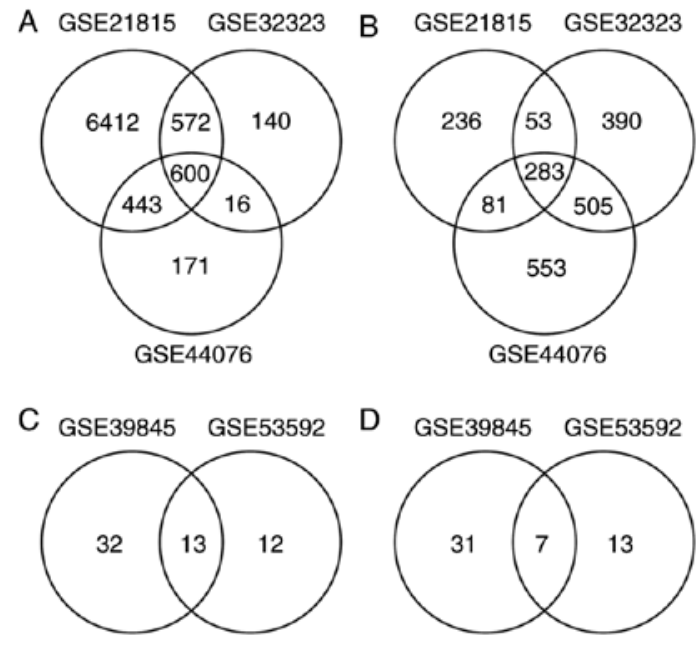

Figure 1. Identification of DEGs and DE miRNAs. (A) Identification of upregulated DEGs. (B) Identification of downregulated DEGs. (C) Identification of upregulated DE miRNAs. (D) Identification of downregulated DE miRNAs.

Clinical Research Ethics Committee of The First Affiliated Hospital of Chongqing Medical University. Informed consents were signed by all patients for the acquisition and use of tissue samples.

Quantitative real-time PCR. Total RNA was extracted from cultured cells (HCT116 and HT-29) by RNAiso Plus (Takara Bio, Dalian, China) and tissues by TRIzol reagent (Invitrogen, Carlsbad,CA, USA). RNA (1 $\mu \mathrm{g})$ was reverse transcribed by the GoScript $^{\mathrm{TM}}$ Reverse Transcription system (Promega, Madison, WI, USA). Real-time PCR was performed by the GoTaq qPCR Master Mix (Promega). All q-PCR values of each gene were normalized against ACTB. The relative expressions of eight DEGs were calculated by the $2^{-\Delta \Delta C t}$ method (15).

Statistical analysis. The final data were presented as mean $\pm \mathrm{SD}$. The results were analyzed by the Student's t-test, and $\mathrm{P}<0.05$ was considered to show statistical difference.

\section{Results}

Identification of DEGs. A total of 8027, 1328 and 1230 upregulated DEGs were identified with the 653,1231 and 1422 downregulated DEGs from GSE21815, GSE32323 and GSE44076, respectively. There were 600 upregulated DEGs (Fig. 1A) and 283 downregulated DEGs (Fig. 1B) in the overlap of the three datasets.

Functional enrichment analysis of DEGs. KOBAS 3.0 was performed to analyze the functional and pathway enrichment of identified DEGs. GO analysis results showed that upregulated DEGs were significantly enriched in binding, protein binding and organic cyclic compound binding at MF level; organelle, intracellular part and cell part at CC level and cellular process, metabolic process and organic substance metabolic process at BP level (Fig. 2). Downregulated DEGs were enriched in binding, receptor binding and protein binding at MF level; extracellular region, cell periphery and plasma 


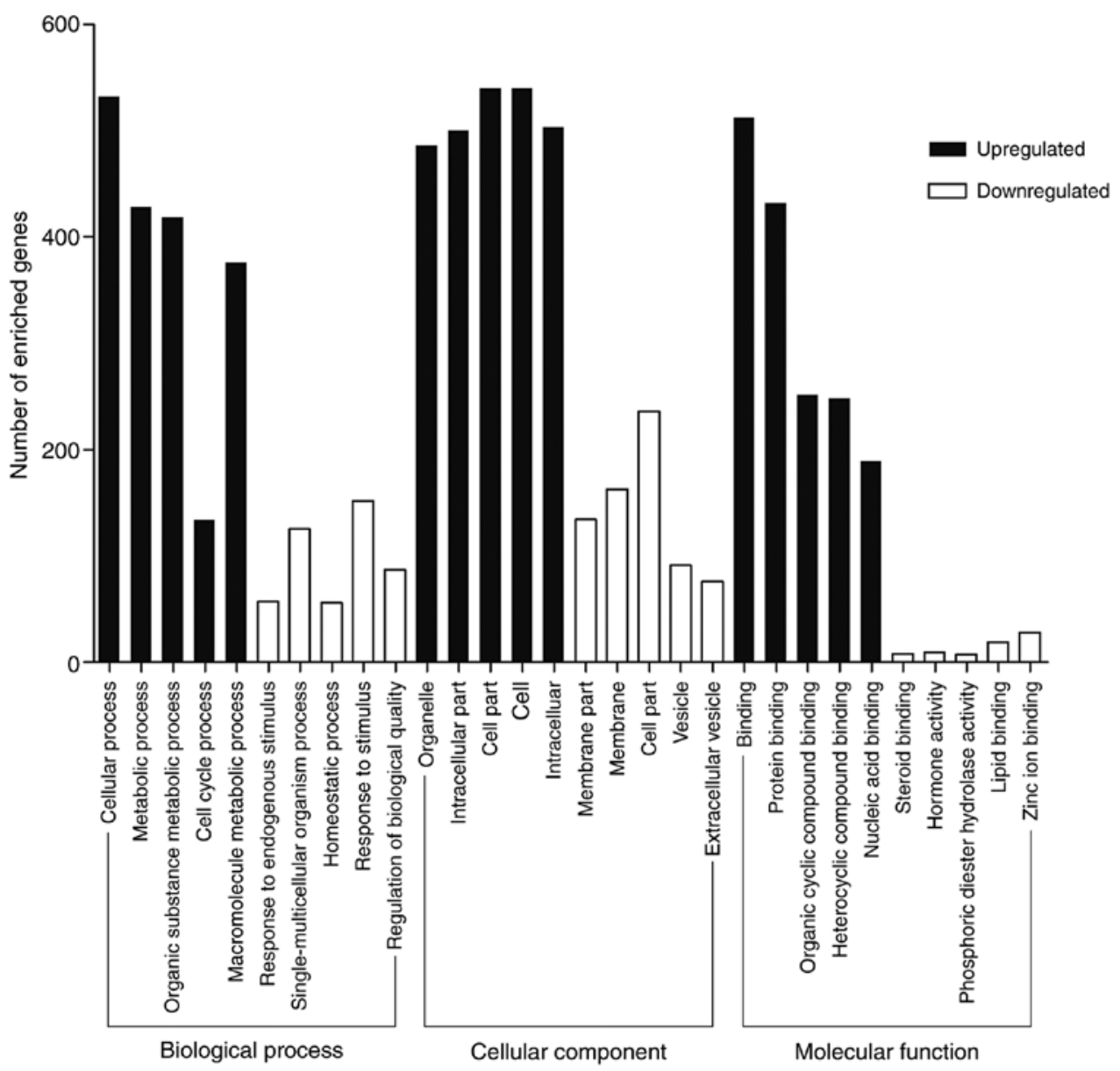

Figure 2. GO analysis of the DEGs. Black bars stand for the upregulated DEGs, while white bars stand for the downregulated DEGs.
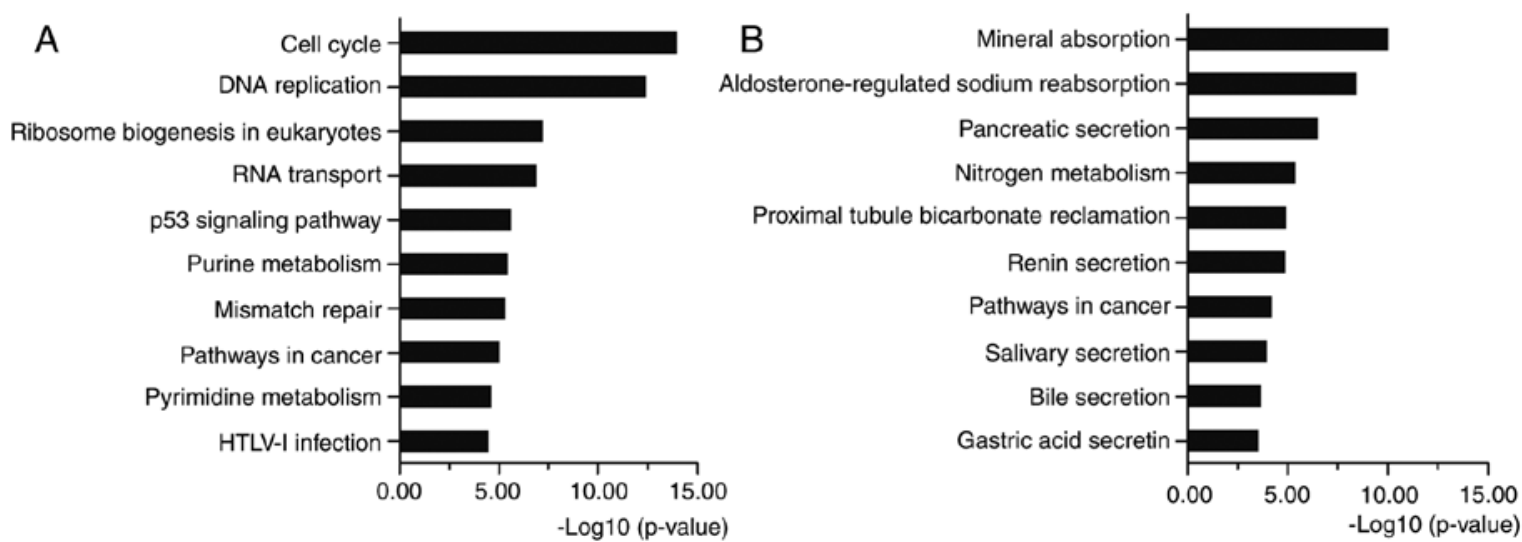

Figure 3. KEGG pathway analysis of DEGs. (A) KEGG pathway analysis of upregulated DEGs. (B) KEGG pathway analysis of downregulated DEGs.

membrane at CC level and chemical homeostasis, cellular response to chemical stimulus and single-organism process at BP level (Fig. 2). Most GO terms enriched in the regulation processes. Binding is a basic step of regulation process. The GO terms mostly enriched in binding which is consistent with the knowledge that tumor development and progression is regulated by multiple molecules (16-18). The GO analysis supported the correlation between the identified DEGs and CRC.

KEGG analysis showed that the upregulated DEGs were mostly enriched in cell cycle, DNA replication, p53 signaling pathway and pathways in cancer (Fig. 3A). The downregulated DEGs were enriched in metabolic pathways and pathways in cancer (Fig. 3B). Cell cycle is the series of events that take place in a cell leading to its division and DNA replication to produce two new cells. Abnormal cell cycle regulation will lead to the occurrence of cancer. Many molecules participate in cancers by regulating the cell cycle $(19,20)$. Multiple genes can disturb DNA replication and cause DNA replication stress and genome instability (21). Oncogenes increase DNA replicative stress and induce a DNA damage response early in tumorigenesis (22). Activated by various factors, p53 


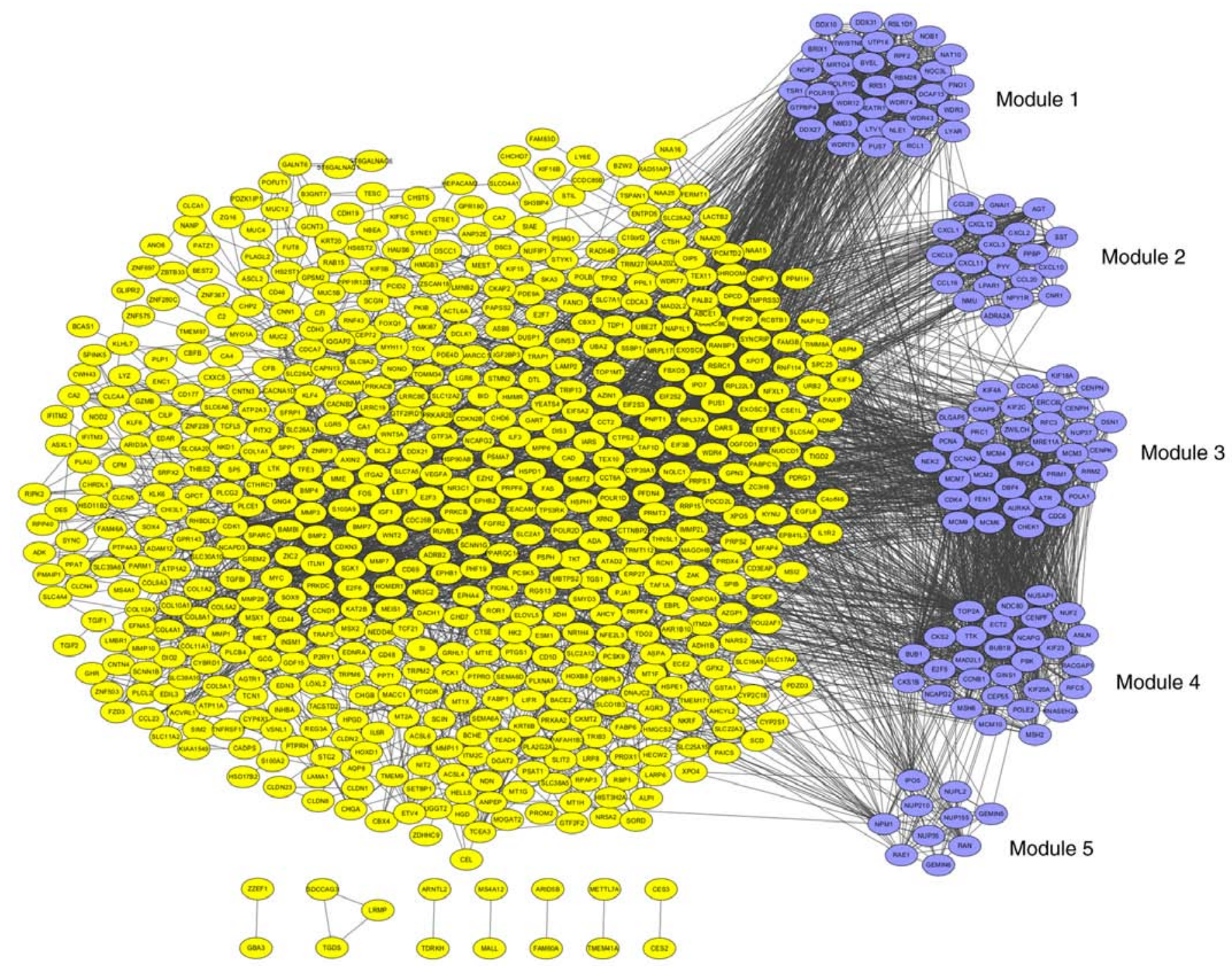

Figure 4. PPI network construction and module analysis. Yellow nodes represent the DEGs, purple nodes represent the genes involved in modules and the lines represent the interaction between two nodes.

signaling pathway represses cancer progression by arresting growth, or by promoting cellular death programs (23). Various molecules regulate the cell cycle and apoptosis though activating or repressing p53 signaling pathway (24-26). Pathways in cancer consist of many classical signaling pathways, such as Wnt, PI3K-Akt, MAPK, VEGF, PPAR, TGF- $\beta$ and p53 signaling pathways, which play significant roles in regulating cell proliferation, apoptosis, invasion, metastasis and adhesion. The KEGG pathway enrichment proved the relationship between the identified DEGs and CRC. All the above analyses supported the reliability of the results in the present study.

PPI network construction and analysis of modules. The PPI network was constructed by STRING and visualized by Cytoscape (version 3.4.0) (Fig. 4). Degree $\geq 20$ was set as the cut-off criterion. A total of 168 DEGs were chosen as hub genes, such as CAD, ITGA2, BCL2, PRKACB, IGF1, SGK1, E2F3 and NR3C1. Moreover, the whole PPI network was analyzed by MCODE. The top five modules were selected, and the KEGG pathway enrichment analysis of the genes involved in modules were performed by KOBAS 3.0. The pathway enrichment analysis revealed that DEGs in these modules

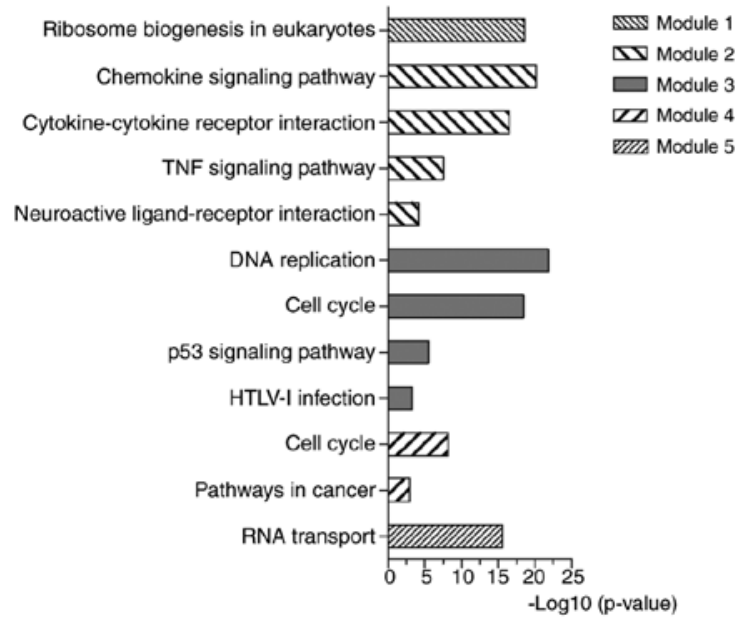

Figure 5. KEGG pathway analysis of the genes in the top five modules.

were mostly enriched in TNF signaling pathway, DNA replication, cell cycle, p53 signaling pathway and pathways in cancer (Fig. 5). 


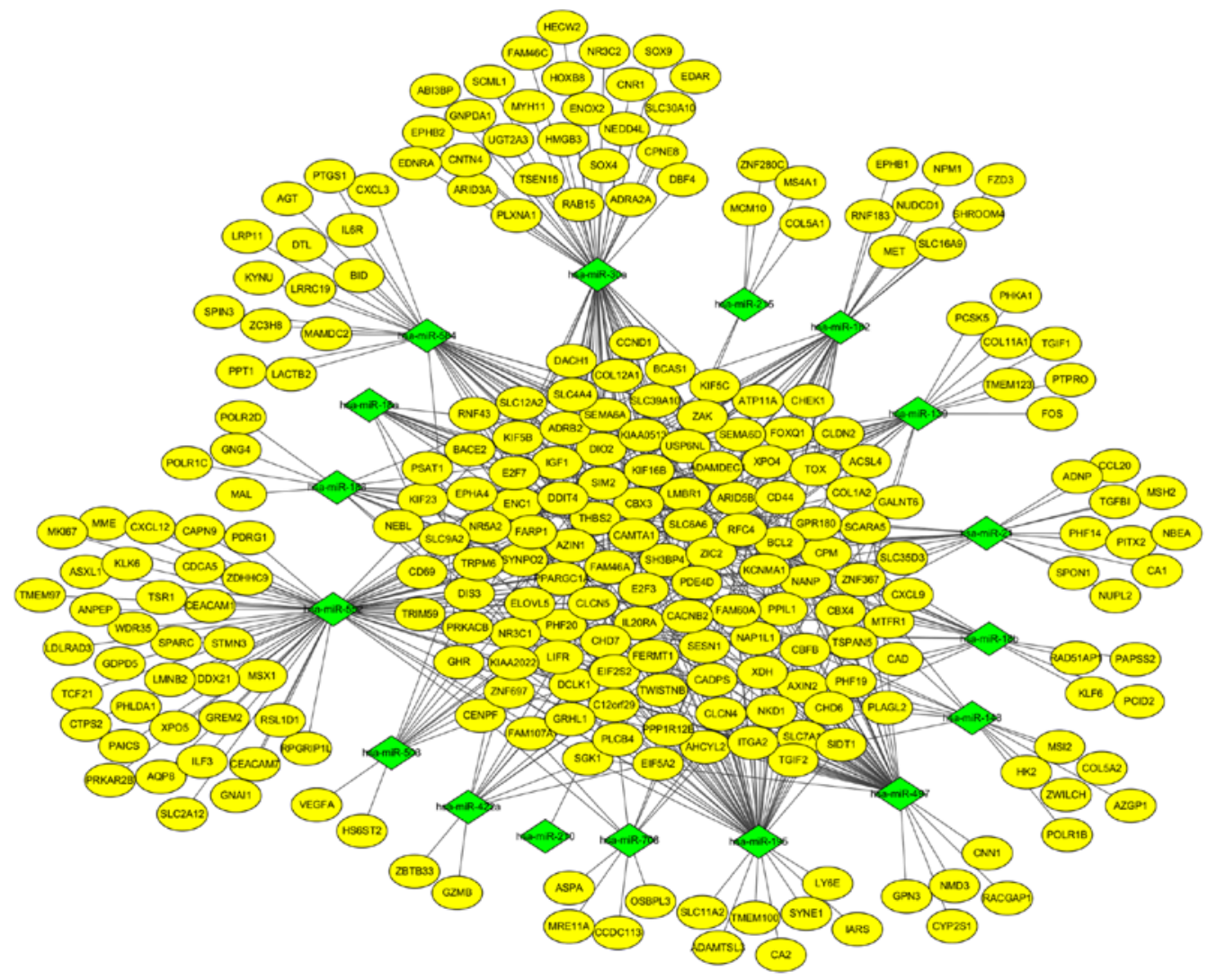

Figure 6. miRNA-gene network. Yellow nodes stand for DEGs, while green nodes stand for DE miRNAs. The lines stand for the regulation relationship between DE miRNAs and DEGs.

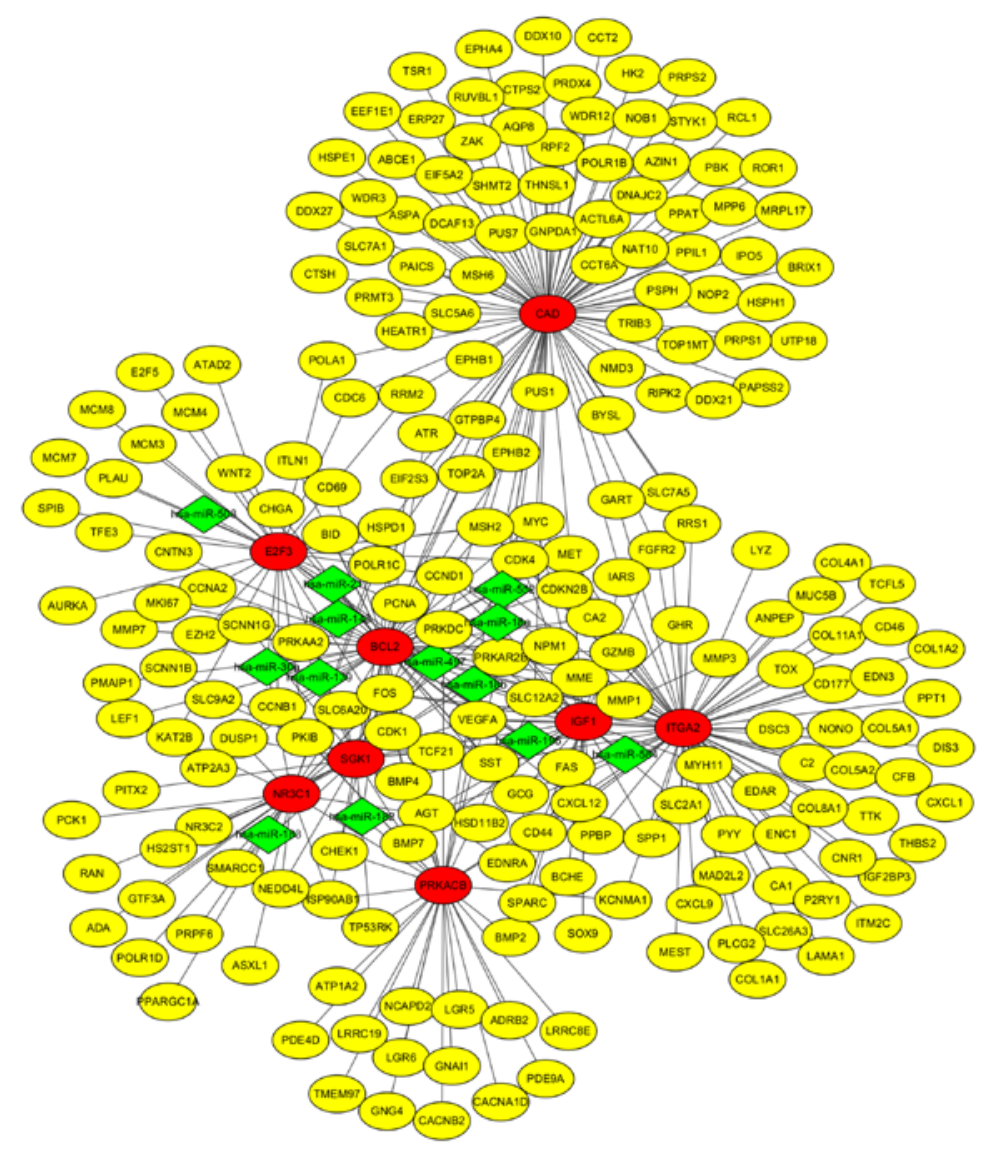

Figure 7. Eight gene biomarker regulation networks. Yellow nodes stand for DEGs, red nodes stand for the eight gene biomarkers and green nodes stand for DE miRNAs. The lines stand for the regulation relationship between two nodes. 

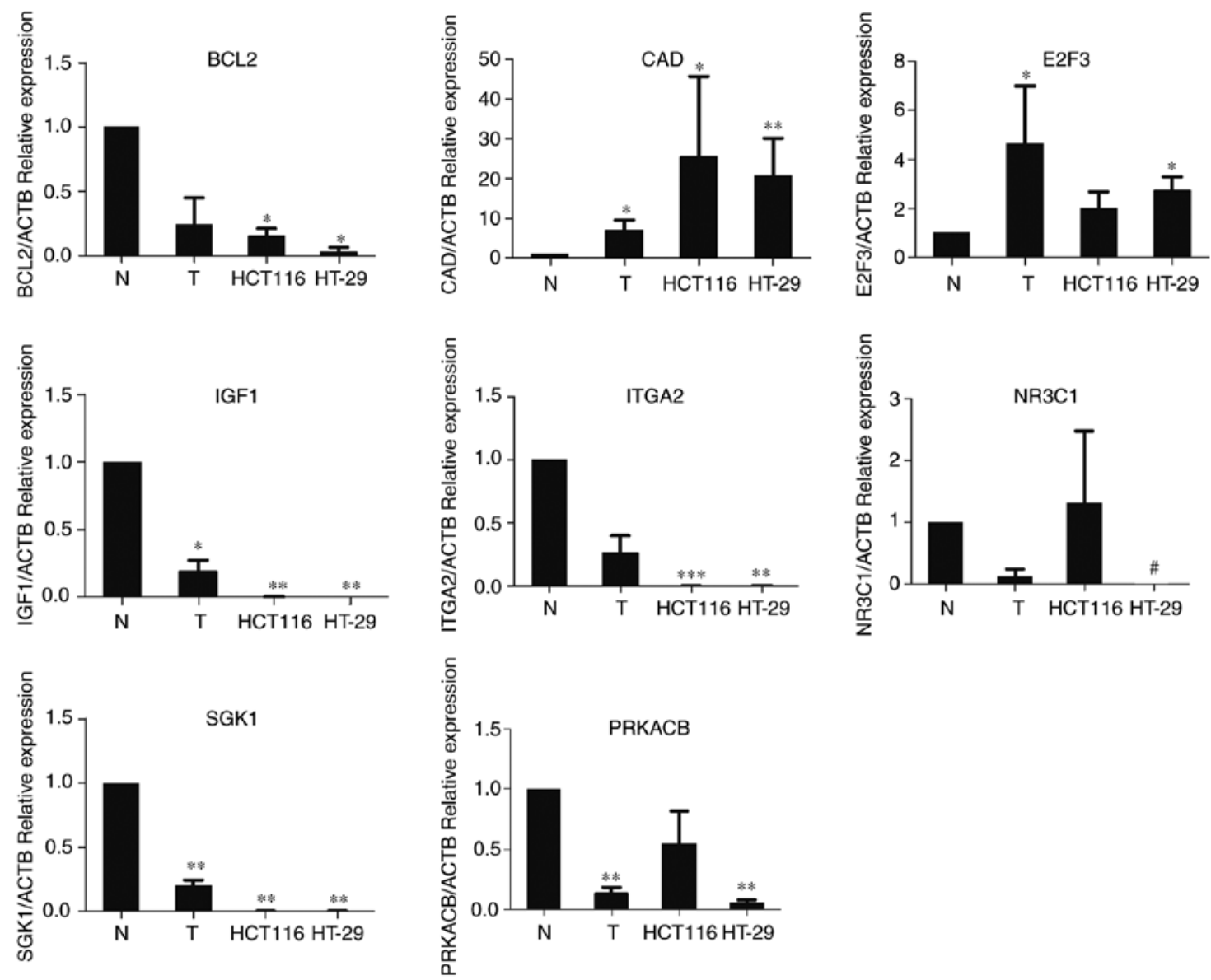

Figure 8. Quantitative real-time PCR results for identified gene biomarkers. Eight candidate genes were selected for further quantitative real-time PCR. Expression of these DEGs was normalized against ACTB expression. The statistical significance of differences was calculated by the Student's $t$-test. " $\mathrm{P}<0.05$, ${ }^{* *} \mathrm{P}<0.01,{ }^{* * *} \mathrm{P}<0.001$. ${ }^{\#}$ The expression of gene could not be detected by $\mathrm{q}-\mathrm{PCR}$.

miRNA-gene network. To further understand the regulatory relationship between identified DEGs and miRNAs, two miRNA profiles were analyzed. A total of 45 and 25 upregulated DE miRNAs (Fig. 1C) were identified with 38 and 20 downregulated DE miRNAs (Fig. 1D) from GSE39845 and GSE53592, respectively. Among them, 13 upregulated DE miRNAs and seven downregulated DE miRNAs were screened out in both datasets. The targeted genes were predicted by the five programs. Besides, to identify the reliable target genes, we compared the targets with DEGs. Only the overlapping genes were selected. Following, miRNA-gene network was constructed (Fig. 6). By analyzing PPI and miRNA-gene network, a total of eight genes were chosen, consisting of 3 upregulated DEGs (CAD, ITGA2 and E2F3) and 5 downregulated DEGs (BCL2, PRKACB, IGF1, SGK1, and NR3C1) (Fig. 7).

Experimental validation of the identified biomarker genes. To verify the bioinformatics analysis for predicting the potential biomarkers in CRC, the eight candidate genes were selected for further quantitative real-time PCR. The expression of eight genes was detected in both tissue samples and CRC cell lines (HCT116 and HT-29). As shown in Fig. 8, seven of the eight identified genes were confirmed to have the same expression trend as predicted by bioinformatics analysis. CAD and E2F3 were overexpressed in tumor tissue samples and CRC cell lines, while the expression of BCL2, PRKACB, IGF1, SGK1 and NR3C1 was reduced in CRC tissue samples and cell lines. However, the expression of ITGA2 in tumor tissue samples and cell lines was lower than that in normal tissue samples. Besides, there was no significant difference in the expression of NR3C1 and PRKACB in HCT116 cell line and normal tissues.

\section{Discussion}

Recently, microarray and bioinformatics analysis have been widely used in identifying potential targets for diagnosis and therapy of different cancers. In the present study, a total of 883 DEGs were screened, including 600 upregulated DEGs and 283 downregulated DEGs. We then performed functional enrichment analysis on the DEGs. For the upregulated DEGs, GO terms were significantly enriched in binding at MF level, organelle at CC level and cellular process at BP level. For the downregulated DEGs, most enriched GO terms were binding at MF level, extracellular region part at $\mathrm{CC}$ level and chemical homeostasis at BP level. Most of these GO terms were basic regulatory concepts in cell. Pathway enrichment analysis revealed that cell cycle, p53 signaling pathway and pathways in cancer were significantly enriched, which were associated with the occurrence of cancers. In addition, we constructed the PPI network and analyzed the top five modules. KEGG pathway 
analysis revealed that the DEGs in these modules were also enriched in cell cycle, p53 signaling pathway and pathways in cancer, which confirmed the findings above. These enriched pathways revealed insight into the molecular mechanisms of $\mathrm{CRC}$, which could provide effective therapeutic strategies for CRC.

miRNAs have been reported to participate in the occurrence and progress of cancer. In the present study, we identified 13 upregulated miRNAs and seven downregulated miRNAs. We then constructed the miRNA-gene network. To identify the key biomarkers of CRC, we combined the two networks. A gene with higher degree in PPI network and regulated by more miRNAs in miRNA-gene network was considered to play a more important role in CRC. A total of eight genes were identified, consisting of three upregulated DEGs (CAD, ITGA2 and E2F3) and five downregulated DEGs (BCL2, PRKACB, IGF1, SGK1 and NR3C1). Besides, hsa-miR-552 and hsa-miR-30a represented the more important role in regulating target genes.

Experimental validation is necessary to confirm our results predicted by bioinformatics analysis. We found that seven of the eight candidate genes have the same expression trend as predicted by qPCR $(\mathrm{P}<0.05)$. Although ITGA2 did not overexpress in $\mathrm{CRC}$ tissue as predicted, it has been reported to be downregulated in prostate cancer (27). In addition, Ferraro et al (28) proved that depressed ITGA2 could reduce cell migration in colon cancer. As for NR3C1, its expression in HCT116 cell line was significantly higher than that in tumor tissues, but could not be detected in HT-29 cell line. Lind et al (29) reported that hypermethylation was significantly associated with the absence or reduced expression of $\mathrm{NR} 3 \mathrm{C} 1$, which leads to different expression levels of NR3C1 in different tissues and cells.

The pathogenesis of cancer is a complex process driven by many factors, including genetic and epigenetic alterations. There was no report on CAD in CRC. However, increased expression of CAD was associated with local tumor extension and cancer recurrence in prostate cancer (30). It may become a novel biomarker of CRC. Downregulation of E2F3 could inhibit cell proliferation and induce apoptosis in CRC. Besides, E2F3 is a target gene of several miRNAs $(31,32)$. BCL2 is an apoptosis regulator. It has been identified as a cause of several cancers, including CRC, melanoma, breast and lung cancer (33-36). PRKACB is a member of the serine/threonine protein kinase family and encodes a catalytic subunit of cAMP-dependent protein kinase. Kvissel et al (37) reported that PRKACB isoforms play different roles in proliferation and differentiation in prostate cancer. IGF1 polymorphisms could influence the risk of CRC (38). For example, the SNP rs6214 of IGF1 could increase the CRC risk (39). Besides, the expression level of IGF1 correlates significantly with tumor size $(\mathrm{P}=0.0024)$ and depth of invasion $(\mathrm{P}=0.0147)$ in CRC (40). SGK1 promotes survival, invasiveness, and metastasis of CRC cells (41). It can also enhance the growth and migration of NSCLC cells by activating $\beta$-catenin/TCF signaling pathway (42). SGK1 expression is also increased in endometrial cancer. Suppression of the expression of SGK1 can induce autophagy and apoptosis (43). hsa-miR-552 and hsa-miR-30a were identified as key miRNAs in the miRNA-gene network. Downregulation of miR-552 reduced cell proliferation, migration and clonogenicity in CRC (44). Conversely, inhibition of miR-30a promoted cell proliferation, migration and invasion in CRC (45).

Cancer is a complex disease caused by multiple factors. A single reason is not sufficient to explain the mechanism of cancer. Combination of mRNAs, miRNAs and interaction networks can help us to investigate and explain the molecular mechanism of cancer (46). It is an effective approach to identify biomarkers of various diseases.

In the present study, we applied bioinformatics analysis to identify the biomarkers of CRC and performed experimental validation. But there were still some limitations in this study. First, the amount of CRC tissue samples was not enough. Because of the difficulty to obtain clinical samples and information. Second, we only checked two cell lines, which cannot fully reveal the difference in expression of biomarkers in CRC.

In conclusion, eight genes and two miRNAs were identified as the biomarkers of CRC. Besides, this study also provided a series of significant pathways and mechanisms for diagnosis and therapy. Eight genes were verified by experiments, including CAD, ITGA2, E2F3, BCL2, PRKACB, IGF1, SGK1 and $\mathrm{NR} 3 \mathrm{C} 1$, which might become the new stars in research of CRC.

\section{References}

1. Haraldsdottir S,EinarsdottirHM,Smaradottir A, Gunnlaugsson A and Halfdanarson TR: Colorectal cancer - review. Laeknabladid 100: 75-82, 2014 (In Icelandic).

2. Fearon ER: Molecular genetics of colorectal cancer. Annu Rev Pathol 6: 479-507, 2011.

3. Simon K: Colorectal cancer development and advances in screening. Clin Interv Aging 11: 967-976, 2016.

4. Thomas J, Ohtsuka M, Pichler M and Ling H: MicroRNAs: Clinical relevance in colorectal cancer. Int J Mol Sci 16: 28063-28076, 2015.

5. Ren A, Dong Y, Tsoi $\mathrm{H}$ and $\mathrm{Yu}$ J: Detection of miRNA as non-invasive biomarkers of colorectal cancer. Int J Mol Sci 16: 2810-2823, 2015.

6. Ng EK, Chong WW, Jin H, Lam EK, Shin VY, Yu J, Poon TC, Ng SS and Sung JJ: Differential expression of microRNAs in plasma of patients with colorectal cancer: A potential marker for colorectal cancer screening. Gut 58: 1375-1381, 2009.

7. Asangani IA, Rasheed SA, Nikolova DA, Leupold JH, Colburn NH, Post S and Allgayer H: MicroRNA-21 (miR-21) post-transcriptionally downregulates tumor suppressor Pdcd4 and stimulates invasion, intravasation and metastasis in colorectal cancer. Oncogene 27: 2128-2136, 2008.

8. Thomas PD: The Gene Ontology and the meaning of biological function. Methods Mol Biol 1446: 15-24, 2017.

9. Xing Z, Chu C, Chen L and Kong X: The use of Gene Ontology terms and KEGG pathways for analysis and prediction of oncogenes. Biochim Biophys Acta 1860: 2725-2734, 2016.

10. Khunlertgit $\mathrm{N}$ and Yoon BJ: Incorporating topological information for predicting robust cancer subnetwork markers in human protein-protein interaction network. BMC Bioinformatics 17 (Suppl 13): 351, 2016.

11. Clough E and Barrett T: The Gene Expression Omnibus Database. Methods Mol Biol 1418: 93-110, 2016.

12. Xie C, Mao X, Huang J, Ding Y, Wu J, Dong S, Kong L, Gao G, Li CY and Wei L: KOBAS 2.0: A web server for annotation and identification of enriched pathways and diseases. Nucleic Acids Res 39 (Web Server issue): W316-W322, 2011.

13. Kanehisa M, Sato Y, Kawashima M,Furumichi M and Tanabe M: KEGG as a reference resource for gene and protein annotation. Nucleic Acids Res 44 (D1): D457-D462, 2016.

14. Killcoyne S, Carter GW, Smith J and Boyle J: Cytoscape: A community-based framework for network modeling. Methods Mol Biol 563: 219-239, 2009.

15. Livak KJ and Schmittgen TD: Analysis of relative gene expression data using real-time quantitative PCR and the 2(-Delta Delta C(T)) method. Methods 25: 402-408, 2001. 
16. Dashwood MR and Loizidou M: Determination of cell-specific receptor binding using a combination of immunohistochemistry and in vitro autoradiography: Relevance to therapeutic receptor targeting in cancer. Methods Mol Biol 878: 137-147, 2012.

17. de Lacerda TC, Costa-Silva B, Giudice FS, Dias MV de Oliveira GP, Teixeira BL, Dos Santos TG and Martins VR: Prion protein binding to HOP modulates the migration and invasion of colorectal cancer cells. Clin Exp Metastasis 33: 441-451, 2016.

18. Tsujitani S, Saito H, Honboh T, Ataka M, Tanida T, Makino M and Ikeguchi M: Prognostic significance of receptor-binding cancer antigen expressed on SiSo cells (RCAS1) expression in relation to cadherin expression in patients with colorectal carcinoma. Dis Colon Rectum 50: 1241-1249, 2007.

19. Liang B, Liu Z, Cao Y, Zhu C, Zuo Y, Huang L, Wen G, Shang N, Chen Y, Yue X, et al: MC37, a new mono-carbonyl curcumin analog, induces $\mathrm{G} 2 / \mathrm{M}$ cell cycle arrest and mitochondriamediated apoptosis in human colorectal cancer cells. Eur J Pharmacol 796: 139-148, 2017.

20. Chen X, Wu X, Ouyang W, Gu M, Gao Z, Song M, Chen Y, Lin Y, Cao Y and Xiao H: Novel ent-Kaurane diterpenoid from Rubus corchorifolius L. f. inhibits human colon cancer cell growth via inducing cell cycle arrest and apoptosis. J Agric Food Chem 65: 1566-1573, 2017.

21. Boyer AS, Walter D and Sørensen CS: DNA replication and cancer: From dysfunctional replication origin activities to therapeutic opportunities. Semin Cancer Biol 37-38: 16-25, 2016.

22. Hills SA and Diffley JF: DNA replication and oncogene-induced replicative stress. Curr Biol 24: R435-R444, 2014.

23. Stegh AH: Targeting the p53 signaling pathway in cancer therapy - the promises, challenges and perils. Expert Opin Ther Targets 16: 67-83, 2012.

24. Liu YH, Liu GH, Mei JJ and Wang J: The preventive effects of hyperoside on lung cancer in vitro by inducing apoptosis and inhibiting proliferation through caspase-3 and P53 signaling pathway. Biomed Pharmacother 83: 381-391, 2016.

25. Chen J, Wei Y, Feng Q, Ren L, He G, Chang W, Zhu D, Yi T, Lin Q, Tang W, et al: Ribosomal protein S15A promotes malignant transformation and predicts poor outcome in colorectal cancer through misregulation of p53 signaling pathway. Int J Oncol 48: $1628-1638,2016$

26. Xiao S, Zhou Y, Yi W, Luo G, Jiang B, Tian Q, Li Y and Xue M: Fra-1 is downregulated in cervical cancer tissues and promotes cervical cancer cell apoptosis by p53 signaling pathway in vitro. Int J Oncol 46: 1677-1684, 2015.

27. Shaikhibrahim Z, Lindstrot A, Buettner R and Wernert N: Analysis of laser-microdissected prostate cancer tissues reveals potential tumor markers. Int J Mol Med 28: 605-611, 2011.

28. Ferraro A, Boni T and Pintzas A: EZH 2 regulates cofilin activity and colon cancer cell migration by targeting ITGA2 gene. PLoS One 9: e115276, 2014

29. Lind GE, Kleivi K, Meling GI, Teixeira MR, Thiis-Evensen E, Rognum TO and Lothe RA: ADAMTS1, CRABP1, and NR3C1 identified as epigenetically deregulated genes in colorectal tumorigenesis. Cell Oncol 28: 259-272, 2006.

30. Morin A, Fritsch L, Mathieu JR, Gilbert C, Guarmit B, Firlej V, Gallou-Kabani C, Vieillefond A, Delongchamps NB and Cabon F: Identification of CAD as an androgen receptor interactant and an early marker of prostate tumor recurrence. FASEB J 26: 460-467, 2012

31. Chang SW, Yue J, Wang BC and Zhang XL: miR-503 inhibits cell proliferation and induces apoptosis in colorectal cancer cells by targeting E2F3. Int J Clin Exp Pathol 8: 12853-12860, 2015.
32. Fang Y, Gu X, Li Z, Xiang J and Chen Z: miR-449b inhibits the proliferation of SW1116 colon cancer stem cells through downregulation of CCND1 and E2F3 expression. Oncol Rep 30: 399-406, 2013

33. Tong Z, Liu N, Lin L, Guo X, Yang D and Zhang Q: miR-125a-5p inhibits cell proliferation and induces apoptosis in colon cancer via targeting BCL2, BCL2L12 and MCL1. Biomed Pharmacother 75: 129-136, 2015.

34. Kaluzki I, Hrgovic I, Hailemariam-Jahn T, Doll M, Kleemann J, Valesky EM, Kippenberger S, Kaufmann R, Zoeller N and Meissner M: Dimethylfumarate inhibits melanoma cell proliferation via p21 and p53 induction and bcl-2 and cyclin B1 downregulation. Tumour Biol 37: 13627-13635, 2016.

35. Bhushann Meka P, Jarjapu S, Vishwakarma SK, Nanchari SR, Cingeetham A, Annamaneni S, Mukta S, Triveni B and Satti V: Influence of BCL2-938 C>A promoter polymorphism and BCL2 gene expression on the progression of breast cancer. Tumour Biol 37: 6905-6912, 2016.

36. Yang X, Gao F, Ma F, Ren Y, Chen H, Liang X, Han S, Xiong X, Pan W, Zhou C, et al: Association of the functional BCL-2 rs2279115 genetic variant and small cell lung cancer. Tumour Biol 37: 1693-1698, 2016.

37. Kvissel AK, Ramberg H, Eide T, Svindland A, Skålhegg BS and Taskén KA: Androgen dependent regulation of protein kinase A subunits in prostate cancer cells. Cell Signal 19: 401-409, 2007.

38. Wong HL, Koh WP, Probst-Hensch NM, Van den Berg D, Yu MC and Ingles SA: Insulin-like growth factor-1 promoter polymorphisms and colorectal cancer: A functional genomics approach. Gut 57: 1090-1096, 2008.

39. Feik E, Baierl A, Hieger B, Führlinger G, Pentz A, Stättner S, Weiss W, Pulgram T, Leeb G, Mach K, et al: Association of IGF1 and IGFBP3 polymorphisms with colorectal polyps and colorectal cancer risk. Cancer Causes Control 21: 91-97, 2010.

40. Shiratsuchi I, Akagi Y, Kawahara A, Kinugasa T, Romeo K, Yoshida T, Ryu Y, Gotanda Y, Kage $M$ and Shirouzu K: Expression of IGF-1 and IGF-1R and their relation to clinicopathological factors in colorectal cancer. Anticancer Res 31: 2541-2545, 2011

41. Lang F, Perrotti N and Stournaras C: Colorectal carcinoma cells -regulation of survival and growth by SGK1. Int J Biochem Cell Biol 42: 1571-1575, 2010.

42. Xiaobo Y, Qiang L, Xiong Q, Zheng R, Jianhua Z, Zhifeng L, Yijiang $S$ and Zheng J: Serum and glucocorticoid kinase 1 promoted the growth and migration of non-small cell lung cancer cells. Gene 576: 339-346, 2016

43. Conza D, Mirra P, Calì G, Tortora T, Insabato L, Fiory F, Schenone S, Amato R, Beguinot F, Perrotti N, et al: The SGK1 inhibitor SI113 induces autophagy, apoptosis, and endoplasmic reticulum stress in endometrial cancer cells. J Cell Physiol: Feb 8, 2017 (Epub ahead of print). https://doi.org/10.1002/jcp.25850.

44. Wang J, Li H, Wang Y, Wang L, Yan X, Zhang D, Ma X, Du Y, Liu X and Yang Y: MicroRNA-552 enhances metastatic capacity of colorectal cancer cells by targeting a disintegrin and metalloprotease 28. Oncotarget 7: 70194-70210, 2016.

45. Zhang Q, Tang Q, Qin D, Yu L, Huang R, Lv G, Zou Z, Jiang XC, Zou C, Liu W, et al: Role of microRNA 30a targeting insulin receptor substrate 2 in colorectal tumorigenesis. Mol Cell Biol 35: 988-1000, 2015.

46. Zeng T, Sun SY, Wang Y, Zhu H and Chen L: Network biomarkers reveal dysfunctional gene regulations during disease progression. FEBS J 280: 5682-5695, 2013. 